\title{
A Case of Dermatofibrosarcoma Protuberans of the Trunk
}

\author{
Senthil Kumar Aiyappan • Upasana Ranga • \\ Anita Samraj • Saveetha Veeraiyan
}

Received: 24 February 2013 /Accepted: 15 March 2013 / Published online: 28 March 2013

(C) Association of Surgeons of India 2013

\begin{abstract}
Dermatofibrosarcoma protuberans is a rare, usually superficial soft tissue sarcoma, which is diagnosed by its typical clinical manifestations. We report a case of histopathologically proved dermatofibrosarcoma protuberans of the left upper posterior trunk in a 35-year-old female for whom complete surgical resection was done.
\end{abstract}

Keywords Dermatofibrosarcoma protuberans $\cdot$ Trunk $\cdot$ CT

\section{Case Summary}

A 35-year-old female patient came to our hospital with history of superficial skin lesion on the left posterior upper trunk region which the patient noticed 10 years back. The lesion was initially small in size and has been progressively mildly increasing in size with a recent sudden increase in size. Presently, the size of the lesion was $11 \times 8 \mathrm{~cm}$, and it was nontender, firm, well defined, and flesh colored, with an irregular surface (Fig. 1). Clinically, diagnosis of dermatofibrosarcoma protuberans was made, and the patient was sent for contrast-enhanced computed tomography (CT) chest examination to look for local extent.

$\mathrm{CT}$ chest showed the presence of an enhancing superficial soft tissue mass measuring $3.7 \times 11 \times 8.3 \mathrm{~cm}$ with a cystic area inferiorly (Fig. 2). Underlying muscles and bones were normal. The patient was taken up for surgery with wide en bloc removal of the tumor with a $3-\mathrm{cm}$ surrounding margin. Postoperative $\mathrm{CT}$ showed complete clearance of the tumor.

S. K. Aiyappan $(\bowtie) \cdot U$. Ranga $\cdot$ S. Veeraiyan Department of Radiodiagnosis and Imaging,

Saveetha Medical College and Hospital, Thandalam,

Kancheepuram, Tamil Nadu 602105, India

e-mail: senthilkumarpgi@yahoo.co.in

\section{A. Samraj}

Department of General Surgery, Saveetha Medical College and Hospital, Thandalam, Kancheepuram,

Tamil Nadu 602105, India
Histopathology was suggestive of dermatofibrosarcoma protuberans with no evidence of fibrosarcomatous component.

\section{Discussion}

Dermatofibrosarcoma protuberans was originally described as a distinct clinicopathologic entity in 1924 by Darier and Ferrand [1]. Microscopically, it is characterized by fibroblasts with a prominent storiform pattern. The name dermatofibrosarcoma protuberans (DFSP) was coined by Hoffman in 1925 [1].

Dermatofibrosarcoma protuberans is an uncommon superficial spindle cell tumor which accounts for about $6 \%$ of all soft tissue sarcomas [1]. The tumor is small and superficial, appears in early adult life, and typically grows slowly over the years. The tumor is firm and fibrous and has a violaceous red color. Pressure on the tumor surface will cause it to blanch [2]. The tumor surface enlarges by expansile growth while the periphery infiltrates the adjacent skin and subcutaneous tissue forming circumferentially located nodules. These nodules coalesce to form a fibrotic plaque in the dermis [2]. Nodular

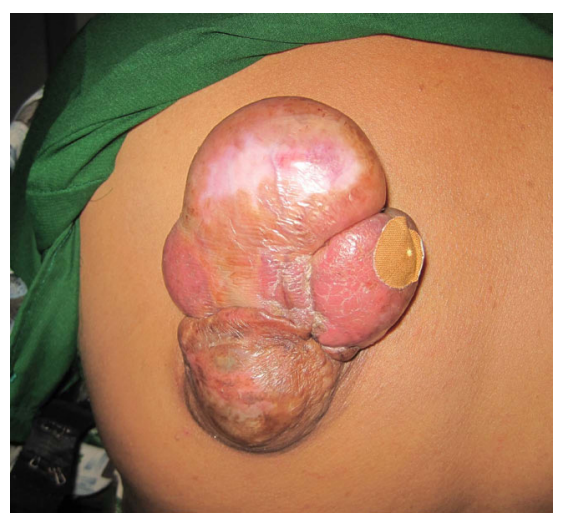

Fig. 1 Clinical image showing a large well-defined flesh-colored lobulated mass in the left posterior upper trunk 
Fig. 2 Axial (a) and sagittal (b) contrast-enhanced CT chest sections show the presence of an enhancing superficial soft tissue mass involving skin and subcutaneous fat (white arrows) of the left upper posterior chest at the level of the scapula. An inferiorly nonenhancing cystic component is seen (black arrow)
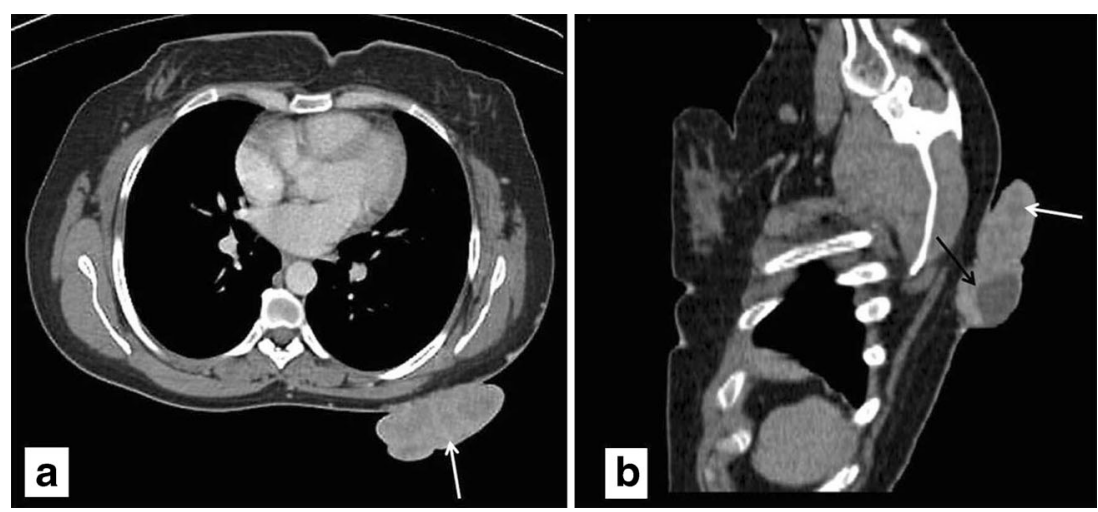

protrusions appear on the surface after a few years, and at this stage, growth frequently accelerates. Due to this, the overlying skin is stretched and undergoes atrophic changes which will lead to increased susceptibility to trauma, forming superficial ulceration and bleeding [2]. Males are slightly more commonly affected, and the highest frequency is reported between the second and the fifth decades [3]. The trunk appears to be the most common site followed by the extremities and the head and neck region [2,3]. Patients often ignore these tumors due to their slow growth. Hence, the imaging appearance of this entity is less reported [1].

The radiologic appearance of dermatofibrosarcoma is that of nodular unmineralized soft tissue mass involving skin and subcutaneous fat $[1,3]$. CT or magnetic resonance imaging images can well show this location, the relation of the lesion to underlying structures, and the distinct lobular or nodular architecture [1,3]. Moderate enhancement can be seen on CT scans after intravenous contrast administration [3, 4].

Histologically, several variants of DFSP have been described which include pigmented (Bednar tumor), myxoid, myoid, granular cell, sclerotic, atrophic DFSP, giant cell fibroblastoma, and DFSP with fibrosarcomatous areas [5]. Out of all these variants, only the DFSP with fibrosarcomatous areas is high grade, with a higher rate of local recurrence and distant metastasis [5]. The fibrosarcomatous variant of DFSP is uncommon and is associated with a significantly more aggressive clinical course indicating a possible need for treatment intensification [6]. Histologically, the fibrosarcomatous component shows presence of focal necrosis and higher mitotic rate [6]. Fibrosarcomatous change is more common in the primary than in recurrent lesions [6].
A wide local excision with a margin of at least $3 \mathrm{~cm}$ and en bloc removal of underlying subcutaneous tissue and fascia is the standard treatment [1]. Mohs micrographic surgery is the treatment of choice with a better cure rate and maximal conservation of tissue [5]. Local recurrence after an apparently adequate surgical excision has been well documented [5]. When surgery is insufficient, imatinib mesylate is a safe and effective treatment in DFSP, especially in cases of locally advanced or metastatic disease [5].

\section{References}

1. Kransdorf MJ, Meis-Kindblom JM (1994) Dermatofibrosarcoma protuberans: radiologic appearance. AJR Am J Roentgenol 163(2):391-394

2. McPeak CJ, Cruz T, Nicastri AD (1967) Dermatofibrosarcoma protuberans: an analysis of 86 cases - five with metastasis. Ann Surg 166(5):803-816

3. Torreggiani WC, Al-Ismail K, Munk PL, Nicolaou S, O'Connell JX, Knowling MA (2002) Dermatofibrosarcoma protuberans: MR imaging features. AJR Am J Roentgenol 178(4):989-993

4. Li X, Zhang W, Xiao L, Zeng Q, Wu X, Qin J, Shen Y (2012) Computed tomographic and pathological findings of dermatofibrosarcoma protuberans. J Comput Assist Tomogr 36(4):462-468

5. Llombart B, Serra-Guillén C, Monteagudo C, López Guerrero JA, Sanmartín O (2013) Dermatofibrosarcoma protuberans: a comprehensive review and update on diagnosis and management. Semin Diagn Pathol 30(1):13-28

6. Mentzel T, Beham A, Katenkamp D, Dei Tos AP, Fletcher CD (1998) Fibrosarcomatous ("high-grade") dermatofibrosarcoma protuberans: clinicopathologic and immunohistochemical study of a series of 41 cases with emphasis on prognostic significance. Am J Surg Pathol 22(5):576-587 